\title{
ON A CONDITION OF OHNISHI
}

\author{
HERBERT A. HOLLISTER
}

In a group $G$, we let $S(x)$ denote the normal subsemigroup generated by $x$ and $S^{\prime}(x)=S(x) \cup e$. Following Fuchs [2], an element $x$ of $G$ is said to be generalized periodic (GP) iff $e \in S(x)$ and nongeneralized periodic (NGP) otherwise. A group is said to be an $O^{*}$ group iff every partial order on $G$ can be extended to a full order.

Ohnishi [9] considered the following group-theoretic condition:

(i) For all $x, y, t$ in $G$, if $x \in S(t)$ and $y \in S(t)$, then $S(x) \cap S(y) \neq \varnothing$. He proved that a group $G$ is an $O^{*}$ group iff every nontrivial element of $G$ is NGP and $G$ satisfies condition (i). In this paper we will study groups which satisfy condition (i) and will also consider two other conditions, one of which is equivalent to (i) and another which is implied by (i).

We remark first that if $G$ satisfies (i), it is known and easily verified that any homomorphic image of $G$ also satisfies (i). Thus a homomorphic image of an $O^{*}$ group is an $O^{*}$ group iff every nontrivial element is NGP.

Theorem 1. If the group $G$ satisfies condition (i), then its GP elements form a normal subgroup $H$ of $G$ and $G / H$ is an $O^{*}$ group.

Proof. Let $x$ and $y$ be GP elements of $G$. Then $\prod_{i=1}^{n} x^{u_{i}}=e$ for some $u_{1}, \cdots, u_{n}$ in $G$. $\prod_{i=1}^{n}(x y)^{u_{i}}=\prod_{i=1}^{n}\left(x^{u_{i}}\right) y_{1}=y_{1}$ where $y_{1} \in S(y)$. Since $e \in S(y)$ and $y_{1} \in S(y)$, condition (i) implies $S(e) \cap S\left(y_{1}\right) \neq \varnothing$ so $e \in S\left(y_{1}\right)$ and $\prod_{j=1}^{m} y_{1} v_{i}=e$ for some $v_{1}, \cdots, v_{m}$ in $G$. Hence $\prod_{j=1}^{m}\left(\prod_{i=1}^{n}(x y)^{u_{i}}\right)^{v_{i}=e}$ and $x y$ is GP. Clearly the inverse and all conjugates of a GP element are GP. If $y \in G-H$, then $S(y) \cap H=\varnothing$ so $G / H$ is NGP. $G / H$ satisfies (i) because $G$ does and therefore $G / H$ is $O^{*}$.

Theorem 2. If $G / N$ is an $O^{*}$ group and every element of $N$ is GP, then $G$ satisfies (i) and $G-N$ is the set of NGP elements of $G$.

Proof. One easily verifies that in $G / N, S(x N)=S(x) N$. Let $t \in G$, $x \in S(t)$, and $y \in S(t)$. If $t \in N$, then $e \in S(x) \cap S(y)$. If $t \notin N$, then $t N$ is NGP in $G / N$. $x N \in S(t N)$ and $y \in S(t N)$ so $S(x N) \cap S(y N) \neq \varnothing$, i.e. $S(x) N \cap S(y) N \neq \varnothing$ so $x_{1} n=y_{1}$ for some $x_{1} \in S(x), n \in N$, and $y_{1} \in S(y)$. Since $n$ is GP, for some $u_{1}, \cdots, u_{m}$ in $G, \prod_{i=1}^{m} n^{u_{i}}=e$. Hence $\prod_{i=1}^{m}\left(x_{1} n\right)^{u_{i}}=\prod_{i=1}^{m}\left(y_{1}\right)^{u_{i}}$ and $x_{2}=x_{2} \prod_{i=1}^{m} n^{u_{i}}=\prod_{i=1}^{m} y_{1}^{u_{i}=y_{2}}$ for some $x_{2} \in S(x)$ and $y_{2} \in S(y)$ so $S(x) \cap S(y) \neq \varnothing . G-N$ is the set

Received by the editors April 7, 1967 and, in revised form, August 12, 1967. 
of GP elements of $G$ because the inverse image of an NGP element must be NGP.

Mal'cev [8] and Fuchs [3] have shown that a torsion-free locally nilpotent group is $O^{*}$.

Theorem 3. A locally nilpotent group satisfies condition (i). In a locally nilpotent group, periodicity is equivalent to generalized periodicity.

Proof. Let $G$ be locally nilpotent and $N$ the set of periodic elements of $G$. It is known (Kurosh [7, Vol. II, pp. 215-216]) that $N$ is a normal subgroup of $G$. $G / N$ is thus a torsion-free locally nilpotent group and hence an $O^{*}$ group. By Theorem 2, $G$ satisfies condition (i) and the NGP elements of $G$ are the elements of $G-N$, i.e. the elements of infinite order.

Kokorin [6] and Fuchs [3] have shown that the direct sum of $O^{*}$ groups is again an $O^{*}$ group. Using this result, we now prove the following:

THEOREM 4. If $H$ and $K$ are groups satisfying condition (i), then $H \times K$ also satisfies (i).

Proof. Let $A$ be the set of GP elements of $H$ and $B$ the GP elements of $K$. Then $A \times B$ is the set of GP elements of $H \times K$. Since any GP group satisfies (i), we may assume that $A \times B \neq H \times K$. By Theorem $1, H / A$ and $K / B$ are $O^{*}$ groups and therefore $H / A$ $\times K / B$ is an $O^{*}$ group. Since $(H \times K) /(A \times B)$ is isomorphic to $H / A \times K / B$, it is an $O^{*}$ group. Since $A \times B$ is the set of GP elements of $H \times K$, Theorem 2 implies that $H \times K$ satisfies (i).

Now consider the following group-theoretic condition:

(ii) For every $x, y, z$ in the group $G$, if $y$ is the product of $n$ conjugates of $x$ and $z$ is the product of $m$ conjugates of $x$, then for some integer $k$, some product of $k m$ conjugates of $y$ is equal to some product of $k n$ conjugates of $z$.

It is clear that condition (ii) implies (i). That (i) implies (ii) follows from Theorem 4 when we consider the special case when one of the groups is the integers.

We include the following group-theoretic lemma which may not be known to the reader.

LemmA 5. If $H$ and $K$ are subsets of the group $G$ such that $H \cup K=G$, then either $H$ generates $G$ or $K$ does.

Proof. Let $H_{0}$ and $K_{0}$ denote the subgroups generated by $H$ and $K$ respectively. If $H_{0} \neq G$, let $x \in G-H_{0}$ and $h \in H_{0}$. Then $x h \notin H_{0}$ so $x h \in K$ and $x^{-1} x h=h \in K_{0}$. Thus $H_{0} \subseteq K_{0}$ and $G=H_{0} \cup K_{0}=K_{0}$. 
A partial order on a group $G$ is said to be directed iff every two elements have an upper bound. Clifford [1] has shown that a partial order is directed iff the positive cone $P$ of $G$ generates $G$.

TheOREM 6. If $G$ satisfies condition (i) and some element of $G$ is NGP, then every partial order of $G$ can be extended to a directed order.

Proof. Let $x$ be an NGP element of $G$ and let $P$ be the cone of a maximal partial order for $G$. If $x \notin P \cup P^{-1}$, then $S(x) \cap P \neq \varnothing$ and $S(x) \cap P^{-1} \neq \varnothing$. Thus some $t \in S(x) \cap(P-e)$ and some $u \in S(x)$ $\cap\left(P^{-1}-e\right)$. By condition (i) we have $S(t) \cap S(u) \neq \varnothing$. Hence $(P-e)$ $\cap\left(P^{-1}-e\right) \neq \varnothing$ so $P \cap P^{-1} \neq e$ which is impossible. Thus $x \in P \cup P^{-1}$. If $N$ denotes the set of GP elements of $G$, we know by Theorem 2 that $N$ does not generate $G$ and therefore $G-N$ generates $G$. Since $G-N$ $=P \cup P^{-1}, P$ generates $G$ and $P$ is the cone of a directed order.

The above proof contains a proof of Ohnishi's result since if every nontrivial element of $G$ is NGP, $G=P \cup P^{-1}$.

CoROllaRy 7. If a locally nilpotent group $G$ is not periodic, then every partial order of $G$ can be extended to a directed order.

Kurosh [7, Vol. II, p. 157] has shown that any torsion-free group can be embedded in a torsion-free group with only two conjugate classes. In such a group, every element is GP so $e \in S(t) \cap S(u)$ for all $t$ and $u$ and condition (i) is satisfied. Since there are torsion-free groups not satisfying (i) we see that a group may satisfy (i) while some of its subgroups may not. An important open question is whether or not every subgroup of an $O^{*}$ group is an $O^{*}$ group, i.e. if $G$ satisfies (i) and $G$ is NGP does every subgroup of $G$ also satisfy (i)?

Consider now the following condition:

(iii) For all $x, t$ in $G$ and $n>0$ :

If $t \in S(x)$, then $e \in S\left(t^{-1}, x^{n}\right)$.

The author has shown [5] that a group $G$ has the property that every partial order can be extended to an isolated partial order iff $G$ is torsion-free and satisfies (iii). Such groups are called $I^{*}$ groups.

Condition (i) implies (iii) for suppose $t \in S(x)$ and $n>0$, then $S(t)$ $\cap S\left(x^{n}\right) \neq \varnothing$ so $e \in S\left(t^{-1}, x^{n}\right)$. That (iii) does not imply (i) follows from the following example and results due to Holland.

Let $A(R)$ denote the group of all order preserving permutations of the real numbers. Let $P=\{g \in A(R): x \leqq x g$ for all $x \in R\}$. It is well known that $P$ is the cone of a lattice-order for $A(R)$. Holland [4, p. 405] has shown that two functions $f$ and $g$ in $A(R)$ are conjugate if there is a one-to-one order preserving function $k$ from $R$ onto $R$ such that $x<x f$ iff $x k<x k g$ and $x>x f$ iff $x k>x k g$. 
If we let $k$ be the identity function we see that for any $g \in A(R)$, $g$ is conjugate to $g^{n}$ for all $n>0$ and also that $g$ is conjugate to $g g^{+}$ where $g^{+}=g \bigvee e$. Let $t \in S(g)$, then $e \in S\left(t^{-1}, g\right)=S\left(t^{-1}, g^{n}\right)$ for all $n>0$ and (iii) is satisfied. Now consider the function $x h=x+\sin x$. By Holland's result, $h$ is conjugate to $h^{-1}$ (let $x k=x+\pi$ ) and so $h$ and $h^{-1}$ are GP. We also have $h$ conjugate to $h h^{+}$and therefore $h^{+}=h^{-1} h h^{+}$is an NGP element which is the product of GP elements which is impossible in a group satisfying (i).

It is not known whether or not the direct sum of groups that satisfy condition (iii) must also satisfy the condition.

The author wishes to express his thanks to J. E. McLaughlin and Charles Holland for their guidance and advice in the preparation of his doctoral dissertation, a portion of which is contained in this paper.

\section{REFERENCES}

1. A. H. Clifford, Partially ordered Abelian groups, Ann. of Math. 41 (1940), 465473.

2. L. Fuchs, Partially ordered algebraic systems, Pergamon Press, London, 1963.

3. - On orderable groups, Proc. Internat. Conf. Theory of Groups, Austral. Nat. Univ., Canberra, August, 1965, New York, 1966.

4. Charles Holland, The lattice-ordered group of automorphisms of an ordered set, Michigan Math. J. 10 (1963), 399-408.

5. H. A. Hollister, Groups in which every maximal partial order is isolated, Proc. Amer. Math. Soc. 19 (1968), 267-269.

6. A. I. Kokorin, Ordering a direct product of ordered groups, Ural. Gos. Univ. Mat. Zap. 4:3 (1963), 95-96. (Russian)

7. A. G. Kurosh, The theory of groups, 2nd ed., Chelsea, New York, 1960.

8. A. I. Mal'cev, On the ordering of groups, Trudy Mat. Inst. Steklov. 38 (1951), 173175. (Russian) 164.

9. M. Ohnishi, On linearization of ordered groups, Osaka Math. J. 2 (1950), 161-

Bowling Green State University 